\title{
DERECHOS HUMANOS, SOCIEDAD DE LA INFORMACIÓN Y SOCIEDAD DE RIESGO
}

\author{
María Lucrecia Rovaletti*
}

\begin{abstract}
Resumen: La irrupción de la informática obliga a replantear el derecho a la intimidad, dada la organización de grandes bancos de datos de carácter personal. Debe añadirse además que la posibilidad del entrecruzamiento de la información contenida en los mismos puede facilitar el control y la manipulación de información sensible.

Estas nuevas tecnologías de información y comunicación (TIC), en continuo desarrollo, se han expandido también a los distintos dominios de salud, incidiendo en todos los actores, tanto usuarios como profesionales, poderes públicos y organismos de seguridad social. Dada la particular estigmatización que conlleva el complejo VIH/SIDA, es preciso exigir severas restricciones en el acceso a estos bancos, pero también normativas internacionales acordes, para que con la "sociedad de la información" no se agudice aún más la "sociedad de riesgo".
\end{abstract}

Palabras clave: tecnologías de información y comunicación, derechos personalísimos, confidencialidad, HIV/SIDA

\section{HUMAN RIGHTS, INFORMATION SOCIETY AND RISK SOCIETY}

Abstract: Online bursting obliges to rethink the right to privacy, given the organization of huge databases of personal character. Furthermore, it must be added that the possibility of intercrossing information content may facilitate the control and manipulation of sensible information.

These new information and communication technologies (ICT), in continuous development, have expanded also to different health fields, affecting all stakeholders, both users and professionals, public powers and social security organisms. Given the specific stigmatization towards HIV/AIDS, requesting several restrictions for access in those banks is needed and also international norms to deal with the "information society", so that "risk society" does not get worse.

Key words: information and communication technologies, personal rights, confidentiality, VIH/AIDS

\section{DIREITOS HUMANOS, SOCIEDADE DA INFORMAÇÁO E SOCIEDADE DE RISCO}

Resumo: A irrupção da informática obriga a reconsiderar o direito à intimidade, dada a organização de grandes bancos de dados de caráter pessoal. Deve-se acrescentar, ademais, que a possibilidade do entrecruzamento da informação contida nestes bancos pode facilitar o controle e a manipulação de informação sensível.

Estas novas tecnologias de informação e comunicação (TIC), em contínuo desenvolvimento, têm se expandido também aos diferentes domínios da saúde, incidindo em todos os atores, seja de usuários e profissionais, poderes públicos e organismos de segurança social. Dada a particular estigmatização que implica o complexo HIV/AIDS, é preciso exigir severas restriçōes no acesso a estes bancos, ademais de normativas internacionais acordes, para que a "sociedade da informação" não agudize ainda mais a "sociedade de risco".

Palavras-chave: tecnologias de informação e comunicação, direitos personalíssimos, confidencialidade, HIV/AIDS

\footnotetext{
* Profesora Titular Consulta de la Universidad de Buenos Aires, Cátedra de Psicología, Ética y Derechos Humanos, Buenos Aires, Argentina Correspondencia: mlrova@psi.uba.ar
} 


\section{Derechos humanos, sociedad de la información y sociedad de riesgo}

"La irrupción de la informática obligó a un replanteo del derecho a la intimidad, por la estructuración de grandes bancos de datos de carácter personal y la posibilidad del entrecruzamiento de la información contenida en los mismos.

En esta dirección (...) debía establecerse que los datos personales referidos a enfermedades deben ser registrados con severas restricciones de acceso por parte de terceros, y en particular aquellas que como el SIDA o la convivencia con VIH tienen una grave potencialidad discriminatoria"(1).

\section{Informática y flujo de información}

El surgimiento de ordenadores personales o computadoras y la centralización de procesos en equipos integrados en redes intra o extranet han generado una serie de fenómenos que no sólo afectan al ámbito informático, sino también a la propia organización de las instituciones.

Estas nuevas tecnologías de información y comunicación (TIC) $(2,3)$, en continuo desarrollo, se han expandido también a los distintos dominios de salud, incidiendo en todos los actores, tanto usuarios como profesionales, poderes públicos y organismos de seguridad social. Ahora bien, el desarrollo creciente de las técnicas de recolección y almacenamiento de datos de carácter personal $(1,4)$ y el acceso a los mismos desde lugares remotos de modo casi instantáneo a través de las redes de comunicación han expuesto la privacidad de las personas a una amenaza potencial. Múltiples registros nos sustraen del anonimato constantemente y si toda esa información se agrupara podría mostrar un cuadro exhaustivo de cada sujeto, facilitando con ello el control y la manipulación del mismo.

Cabría preguntarse entonces si la vida privada no va siendo un espacio en desaparición, en la medida en que deviene cada vez más transparente. Las TIC e Internet se han convertido en un formidable reservorio de informaciones susceptibles de explotaciones infinitas. De allí la fuerte tensión entre las TIC y los "datos personales", es decir, aquellos referidos a una persona física identificada o que pueda serlo directa o indirectamente a través de un número de identificación (por ejemplo, el número de la seguridad social); o bien, a través de uno o de varios elementos que le sean propios (las ini- ciales del apellido y del nombre, con comprobación de informaciones como fecha de nacimiento, municipio de residencia, elementos biométricos, etc.)(5).

"En un contexto internacional marcado por fuertes evoluciones tecnológicas, políticas, jurídicas y económicas, ¿cómo proteger la vida humana en un mundo sin fronteras?", fue el lema de la $30^{\text {a }}$ Conferencia Internacional de Autoridades de Protección de Datos y Privacidad (Estraburgo, 2008).

Por ello, se impone como un desafío actual conciliar del mejor modo posible los imperativos de protección y tutela del individuo con los avances de la sociedad de la información.

\section{La tensión entre los derechos personalísimos y la sociedad del conocimiento: el impacto de la informática}

Desde mediados del siglo XX se advierte una apertura hacia una juridicidad más atenta al bienestar de la persona, y la recepción más dedicada a los llamados "derechos personalísimos"(6), en un tránsito desde el clásico Estado liberal de derecho, al Estado social y democrático de derecho(7)'. Se los denomina de esta manera porque "giran en torno a la persona, por y para ella" (6:196) y tutelan los distintos modos de ser del individuo garantizándole el goce pleno de sus potencias y facultades. A diferencia de los otros derechos de primera generación, que se extienden más allá de la incolumidad del cuerpo (como por ejemplo el derecho a la propiedad), estos acentúan principalmente los derechos a la sacralidad del cuerpo y la persona en general.

Se pueden distinguir en estos derechos tres líneas de orientación: (a) la que se refiere a la integridad física del individuo, como el derecho a la vida y a vivir, el derecho al uso y desarrollo del cuerpo como parte esencial del ser humano, así como también el derecho de morir con dignidad y a la utilización del cadáver; (b) la relacionada con la libertad en el ámbito privado, como la expresión de ideas, la libertad sexual y procreacional, la realización de actos jurídicos y el empleo de fuerza física y espiritual, y (c) la que apunta a la integridad espiritual, como la libertad de conciencia y de religión, el derecho al honor, a la propia imagen (incluye el buen nombre y a la honra), en consonancia con el derecho a

1 E. Pérez Escobar los ubica en el ámbito de los derechos de primera generación. 
la intimidad personal en general y con todo los demás derechos que se desprenden de éste, como el derecho al secreto de particularidades que le son propias, a la reserva en las comunicaciones telefónicas, escritas o verbales, que no tienen como objetivo lo público.

En este sentido, el Derecho debe procurar la tutela de las potencias humanas y fijar ciertos límites al ejercicio de los derechos del sujeto, atendiendo al orden general, pero preservando siempre la dignidad de la persona en el marco de libertad propia de la condición esencial del hombre. Esta protección del ámbito de la privacidad a nivel jurídico configura el "derecho a la intimidad".

Con el desarrollo científico y tecnológico surgen nuevas necesidades humanas relacionadas con la informática y el ciberespacio, las cuales requieren también políticas coherentes para que estos bienes sean reconocidos y protegidos. Son los derechos inherentes a la "sociedad del conocimiento", llamados derechos de cuarta generación(8). En el ejercicio de estos derechos se vinculan ahora otros, como el derecho a la libertad de comunicación, pensamiento y expresión, y de una manera indirecta los "derechos personalísimos", dado que las TIC pueden afectar el derecho a la intimidad, a la honra y a la propiedad intelectual, entre otros.

\section{Las nuevas tecnologías de la información y comunicación y el ámbito de la salud}

En 1997, después de que la UNESCO consagrara un congreso internacional al tema, se acunó el término "infoética". De constitución similar a "bioética", esta noción tiene una autonomía y una especificidad irreductible respecto de aquella, aunque ambas disciplinas y de modo complementario planteen la toma de conciencia ética respecto a los adelantos científicos en la sociedad actual.

Hottois define la "infoética" como el estudio, discusión y resolución de problemas éticos suscitados por la investigación y el desarrollo de las nuevas técnicas electrónicas de recolección, producción, almacenamiento, tratamiento, comunicación y usos diversos de información convertidas en el seno de una sociedad global, llamada de "información"(3:514). Estas tecnologías quedan asociadas también a cuestiones sobre infoeconomía e infopolítica.

En el área de la salud, pueden señalarse tres aplicaciones fundamentales: a) El legajo médico informatizado, que reemplaza el papel y contiene un número de informaciones sobre antecedentes familiares de intervenciones médicas sufridas, resultados de análisis y exámenes diversos, medicamentos prescriptos; eventualmente hábitos de vida, aspectos de la situación profesional, social, económica; estadía en establecimientos sanitarios; afiliaciones a la seguridad social y seguros; además puede almacenar imágenes y sonidos. Este legajo puede ser consultado parcial o totalmente por uno $\mathrm{o}$ varios profesionales de una o varias instituciones de salud a través de una red informática.

b) La Tarjeta de Salud informatizada, comparable a la bancaria, está acompañada de un microprocesador en el cual se almacenan informaciones médicas y administrativas útiles para los médicos, instituciones hospitalarias, organismos de seguridad social, laboratorios y farmacias; tiene funciones administrativas y médicas y eventualmente de urgencia ${ }^{2}$. Esta tarjeta vital constituye un minilegajo médico portátil, pero sobre la cual el interesado no dispone de técnicas que le permitan su lectura. El acceso a la información de la misma puede ser compartido por los profesionales gracias a su Tarjeta Profesional de Salud y sólo parcialmente por otros administrativos que no están sujetos al secreto médico. Este instrumento tiene un uso cada vez más corriente y hasta obligatorio para poder acceder a los servicios de salud.

c) La telemedicina, que se apoya en el legajo electrónico y la Tarjeta de Salud, presta atención y ayuda no sólo a pacientes a distancia, sino también permite la comunicación entre expertos y profesionales. En ciertos casos puede permitir al sujeto realizar una teleconsulta.

De este modo, estas nuevas tecnologías permiten disponer rápida e ilimitadamente de informaciones actualizadas, evitando datos repetidos, y permitiendo una rápida interconsulta entre colegas y especialistas; ayudan a gestionar de modo más eficaz los trámites de salud; resultan de gran ayuda para sujetos que habitan en regiones alejadas y desfavorecidas de cuidados médicos, y facilitan los estudios estadísticos y epidemiológicos, lo cual favorece los sistemas preventivos.

Si bien estas tecnologías no se han generalizado sino a pocos países y todavía en algunos de ellos están

2 Estos microprocesadores se encuentran también en algunos documentos de identidad. 
en etapa piloto, resulta importante analizar algunos planteamientos que ya se han generado alrededor de ellas, las que pueden tener especial incidencia con el complejo VIH/SIDA(9-11).

\section{Informática, confidencialidad y complejo VIH/SIDA}

No cabe duda de que en esta "era de la indiscreción" (12) la medicina viola constantemente la intimidad no sólo con el fin de lograr diagnósticos más precisos a través de sus técnicas, estudios e interrogatorios, sino también a través de los comentarios con otros colegas del área salud que trabajan en esas instituciones. Y estos problemas se potencian cada vez más en la medida en que la información puede circular en el ámbito laboral, de aseguradoras y de gerenciadoras. En efecto, a pesar de los avances que estas estrategias informáticas implican, se presentan algunos riesgos respecto de la "confidencialidad", los cuales pueden verse agravados aún más por los aspectos discriminatorios y estigmatizantes que esta pandemia del VIH/SIDA implica(13-16).

\section{Riesgo de empobrecimiento de la relación terapéutica y confidencialidad}

La multiplicación y extensión de las mediaciones técnicas en las relaciones interpersonales sanitarias puede llevar a que los pacientes limiten y encubran datos personales sensibles, importantes para su tratamiento, ante el temor a la pérdida de confidencialidad. Se aumenta de este modo el riesgo de deshumanización en la relación clínica, ya agudizado por la especialización biomédica, el sistema de expertos y las modalidades de gerenciamiento de salud. El paciente se siente un extraño frente a ese legajo informático, lo cual genera desconfianza y falta de colaboración.

\section{Riesgos de falta de control y de seguridad, casos de abusos y responsabilidad por ilícitos por parte del sistema sanitario en general}

La incontrolabilidad del flujo de datos a través de las redes se ve agravada por la imposibilidad de asignar responsabilidades en caso de faltas de seguridad, fraude o errores, intencionales o no. Existe riesgo de uso abusivo por terceros, quienes pueden tener otros intereses potenciales de naturaleza económica y política, si bien a primera vista no intrínsecamente perjudiciales en sí, y pueden usar y/o explotar esta información sin el consentimiento personal.

\section{Riesgos respecto de la intimidad y privacidad, por el tipo de datos personales colectados}

El derecho a la privacidad y el respeto a la confidencialidad, que regulan también las prácticas asistenciales y que obligan a todo el personal asociado a las mismas, inhabilita que datos personales sanitarios identificables puedan ser usados(17). Por eso debe recurrirse tanto a redes cerradas de trasmisión como a técnicas de criptaje, de anonimización y de códigos restrictivos al acceso. Estas son exigencias que provienen de un imperativo ético y también del sistema jurídico.

\section{Riesgo de inaccesibilidad al sistema informático}

El ciudadano común no tiene siempre control ni seguridad evidente y verificable por él mismo de estos datos digitalizados, sobre todo por la multiplicidad de responsables que pueden acrecentar y acceder a su legajo. Debido al obstáculo técnico que supone no sólo el acceso a estas máquinas informáticas sino a sus códigos y lenguajes, el principal interesado no puede ingresar a su propio legajo y muchas veces encuentra resistencia y escasa voluntad por parte del personal técnico.

\section{El derecho a la protección de datos en una sociedad sin fronteras}

La protección de las personas -asociada a la libertad, dignidad e integridad del sujeto- es inherente a los mismos derechos humanos. En este sentido, en el ámbito de la salud, la digitalización de los datos personales debe ser objetivamente justificada en orden al cuidado del paciente y realizada en su presencia, respetando el consentimiento otorgado con esa expresa finalidad. La conservación de ciertos datos sólo debe mantenerse por el tiempo necesario. Más aún, se requiere que se asegure y se proteja la fiabilidad de los mismos, y que su actualización y su accesibilidad sea una facultad exclusiva de personas competentes y autorizadas. Cuando la utilización autorizada de datos médicos implica la posibilidad de identificar a las personas, el tratamiento debe hacerse según anonimización de los mismos. La rastreabilidad de los datos y la identificación del responsable de cada operación es un imperativo, con el fin de 
que se puedan corregir, evitar y sancionar los errores. Toda base de datos personales o red de comunicación secreta de datos personales debe ser prohibida (Ley de Habeas Data) (17).

Por su parte, el interesado podrá además acceder a los datos personales que le conciernen, corregirlos, completarlos, borrarlos o bloquearlos.

En la ya mencionada $30^{\text {a }}$ Conferencia Internacional de Autoridades de Protección de Datos y Privacidad (Estraburgo, 2008), en su "Resolución relativa a la urgente necesidad de proteger la privacidad en un mundo sin fronteras, y de alcanzar una propuesta conjunta para el establecimiento de estándares internacionales sobre privacidad y protección de datos personales", sus miembros muestran que se han llevado a cabo notorios esfuerzos con vistas a alcanzar estos objetivos. Consideran que, con el crecimiento de la sociedad de la información, el derecho a la protección de datos y a la privacidad es una condición indispensable en una sociedad democrática y liberal, para garantizar el respeto a los derechos humanos, así como la libre circulación de información en una economía de mercado.

La globalización de los intercambios y tratamientos de datos personales, la complejidad de los sistemas informáticos, los potenciales perjuicios derivados de la mala utilización de unas tecnologías cada vez más potentes y el incremento de las medidas de seguridad requieren de una respuesta rápida y adecuada, con vistas a garantizar el respeto a los derechos y libertades fundamentales, y en concreto el derecho a la privacidad.

Las diferencias persistentes en los diferentes países en materia de protección de datos y de respeto de la privacidad, especialmente la ausencia de garantías en muchos Estados, perjudican los intercambios de datos personales y la puesta en práctica de una protección efectiva y global. Por eso, resultan prioritarias reglas internacionales que la garanticen y de un modo uniforme. El reconocimiento de estos derechos pasa por la adopción de un instrumento legislativo universal y vinculante que complemente los diferentes instrumentos existentes y que refuerce la cooperación internacional en torno al tema. Se requiere además que las autoridades pertinentes puedan ejercer sus funciones con completa independencia.

En resumen, que la intimidad se erigiera en los siglos XX y XXI como un derecho subjetivo o personalísimo permitió la consolidación del derecho a la privacidad, no sólo como regulación de relaciones humanas que se deben gestionar libremente, sino incluso frente a la interferencia de particulares. Esta evolución ha tenido profundas consecuencias en el ámbito de la relación clínica. Ya no se trata de que el médico evalúe y determine con su propio criterio cuáles datos e informaciones deben reservarse, sino que los individuos puedan y deban gestionar libremente sus derechos, aquellos inherentes "al hecho de ser personas, ciudadanos, sujetos morales libres”. Será el propio interesado el que disponga qué tratamiento habrá que dar a la información sobre su cuerpo, su salud, etc. La confidencialidad indica, precisamente, los límites que rodean las informaciones sensibles -ya sean reservadas, privadas, íntimas o públicas- e indica también cómo guardarlas y preservarlas.

La particular estigmatización que conlleva el complejo VIH/SIDA ha puesto de manifiesto cuán poco cuidadosos suelen ser algunos médicos y el personal de la salud a la hora de guardar el secreto profesional; a veces, estos datos se divulgan también entre familiares y amigos, y pueden llegar hasta a los medios masivos de comunicación. Olvidando que la historia clínica constituye un documento científico, legal y ético que pertenece al paciente, se llega a pensar que cualquier dato consignado en ella pueda ser difundido sin mayor control por el hecho de estar a disposición para fines terapéuticos y hasta docentes. De allí la necesidad de exigir severas restricciones en el acceso a estos bancos, pero también normativas internacionales acordes para que la "sociedad de la información" no agudice aún más la "sociedad de riesgo"(18-20).

\section{Referencias}

1. Molina Quiroga E. Protección de datos personales (a propósito de la nueva ley 25.326). Portal de abogados. Disponible en: http://www.portaldeabogados.com.ar/derechoinformatico/protecciondatos.htm\#up

2. Bevort Brunder E, Breda I, Fluckiger C, et al. Technologies de l'Information et de la Communication: Construction de Soi et Autonomie. Agora 2007; 46: 14-84. 
3. Hottois G, Missa JN. Nouvelle Encyclopédie de la Bioéthique. Médecine, Environnenment, Biotechnologie. Bruxelles: De Boeck Université; 2001: 315-729.

4. Junta de Castilla y León, Consejería de Sanidad, Dirección General de Planificación y Ordenación. Guía de intimidad, confidencialidad, y protección de datos de carácter personal. Disponible en: http://www.salud.jcyl.es/sanidad/cm/institucion/ tkContent?idContent=718131\&locale=es_ES\&textOnly=false

5. Comité Consultatif National d'Ethique pour les Sciences de la Vie et de la Santé. Le "dossier médical personnel” et l'informatisation des données de santé, Avis 2008; 104: 1-16 Avaible from: www.ccne-ethique.fr/docs/Avis\%20104. pdf

6. Cifuentes S. Derechos personalísimos. Buenos Aires: Editorial Astrea; 1995.

7. Pérez Escobar E. Los Derechos Fundamentales: historia, concepto y fundamento. Barcelona: Cátedra del Máster de Bioética del Instituto Borja de Bioética, Universidad Ramón Lull; 2004.

8. Vasak K. (ed.) The International Dimensions of Human Rights. Paris: UNESCO/Greenwood Press; 1982.

9. Bayer R. Ethics and Public Policy: Engaging the Moral Challenges Posed by AIDS. AIDS patient care and STD's 2006; 20(7): 456-460.

10. Bayer R. Public health policy and the AIDS epidemic. The New England Journal of Medicine 1991; 324(21): 15001504.

11. Dickens BM. Legal limits of AIDS confidentiality. JAMA 1988; 259(23): 3449-3451.

12. Siegler M. Sounding Boards. Confidentiality in medicine: 'a decrepit concept'. The New England Journal of Medicine 1982; 307(24): 1518-1521.

13. Bayer R. La confidencialidad y sus límites. En: Fuenzalida-Puelma H, et al. Aportes de la Etica y el Derecho al estudio del SIDA. Washington: OPS; 1991: 155-157.

14. GABA-BA: Directos Humanos e AIDS. A transversalidade dos directos humanos como referencial ético e político da açâo de GAPA-Bahia. Cuadernos Abong 2000; 28: 131-175.

15. Rovaletti ML. El SIDA: entre la confidencialidad y la coerción. Jurisprudencia Argentina-Lexis Nexis 2008; I: 40-43.

16. Rovaletti ML. La confidencialidad en los tiempos de HIV/SIDA. Jurisprudencia Argentina-Lexis Nexis 2005; IV(5): 60-63.

17. República Argentina. Ley 25.326 de Protección de los datos personales (Habeas Data). Promulgada parcialmente el 3010-2000. Disponible en: http://www.protecciondedatos.com.ar/ley25326.htm

18. Beck U. Modernización reflexiva. Madrid: Alianza Editorial; 1994;

19. Beck U. La sociedad del riesgo. Hacia una nueva modernidad. Barcelona: Paidós; 1994.

20. Giddens A. Consecuencias de la modernidad. Madrid: Alianza Editorial; 1990.

Recibido: 13 de febrero de 2009

Aceptado: 25 de mayo de 2009 